ANNALES

POLONICI MATHEMATICI

$83.1(2004)$

\title{
Natural operators lifting functions to affinors on higher order cotangent bundles
}

\author{
by W. M. Mikulski (Kraków)
}

\begin{abstract}
For natural numbers $n \geq 3$ and $r \geq 1$ all natural operators $A: T_{\mid \mathcal{M} f_{n}}^{(0,0)} \rightsquigarrow$ $T^{(1,1)} T^{r *}$ transforming functions from $n$-manifolds into affinors (i.e. tensor fields of type $(1,1))$ on the $r$-cotangent bundle are classified.
\end{abstract}

Introduction. We study the problem of how a function $f: M \rightarrow \mathbb{R}$ on an $n$-manifold $M$ induces an affinor $A(f)$ on the $r$-cotangent bundle $T^{r *} M=J^{r}(M, \mathbb{R})_{0}$. This problem is reflected in the concept of natural operators $A: T_{\mid \mathcal{M} f_{n}}^{(0,0)} \rightsquigarrow T^{(1,1)} T^{r *}$ in the sense of [3]. We prove that for $n \geq 3$ the space of all natural operators $A: T_{\mid \mathcal{M} f_{n}}^{(0,0)} \rightsquigarrow T^{(1,1)} T^{r *}$ is a free $[(r+1)(r+2)-1]$-dimensional module over $\mathcal{C}^{\infty}(\mathbb{R})$ and we construct explicitly a basis of this module.

In the proof we will use a result of J. Kurek [5] stating that for $n \geq 2$ the space of all natural affinors on $T^{r *}$ over $n$-manifolds is an $(r+1)$-dimensional vector space over $\mathbb{R}$.

Natural affinors can be used to study torsions of connections (see [4]). That is why they have been classified in many papers ([1]-[3], [5]-[7], etc.). It seems that natural affinors depending on some geometric objects (functions, vector fields, forms, etc.) can also be used to study invariants of connections depending on these objects. That is why classifications of affinors depending on some geometric objects are useful.

The usual coordinates on $\mathbb{R}^{n}$ are denoted by $x^{1}, \ldots, x^{n}$, and we write $\partial_{i}=\partial / \partial x^{i}$ for $i=1, \ldots, n$.

All manifolds and maps are assumed to be smooth, i.e. of class $\mathcal{C}^{\infty}$.

1. The $r$-cotangent bundle. For any $n$-dimensional manifold $M$ we have the vector bundle $T^{r *} M=J^{r}(M, \mathbb{R})_{0}=\left\{j_{x}^{r} \gamma \mid \gamma: M \rightarrow \mathbb{R}, x \in M\right.$,

2000 Mathematics Subject Classification: 58A20, 53A55.

Key words and phrases: natural bundles, natural operators. 
$\gamma(x)=0\}$ over $M$. It is called the $r$-cotangent bundle of $M$. Let $\pi: T^{r *} M$ $\rightarrow M$ be the bundle projection. Every embedding $\varphi: M \rightarrow N$ of two $n$-manifolds induces a vector bundle map $T^{r *} \varphi: T^{r *} M \rightarrow T^{r *} N$ over $\varphi$, namely $T^{r *} \varphi\left(j_{x}^{r}(\gamma)\right)=j_{\varphi(x)}^{r}\left(\gamma \circ \varphi^{-1}\right), j_{0}^{r}(\gamma) \in T^{r *} M$. The functor $T^{r *}$ : $\mathcal{M} f_{n} \rightarrow \mathcal{V B}$ is a natural vector bundle over $n$-manifolds [3].

2. Examples of natural operators $T_{\mid \mathcal{M} f_{n}}^{(0,0)} \rightsquigarrow T^{(1,1)} T^{r *}$. A natural operator $A: T_{\mid \mathcal{M} f_{n}}^{(0,0)} \rightsquigarrow T^{(1,1)} T^{r *}$ is an $\mathcal{M} f_{n}$-invariant family of regular operators

$$
A: \mathcal{C}^{\infty}(M) \rightarrow \operatorname{AFF}\left(T^{r *} M\right)
$$

from the algebra $\mathcal{C}^{\infty}(M)$ of smooth maps $M \rightarrow \mathbb{R}$ into the vector space $\operatorname{AFF}\left(T^{r *} M\right)$ of all affinors (tensor fields of type $(1,1)$ ) on $T^{r *} M$ for any $n$-manifold $M$. The $\mathcal{M} f_{n}$-invariance means that for any maps $f_{1}: M \rightarrow \mathbb{R}$ and $f_{2}: N \rightarrow \mathbb{R}$ and any local diffeomorphism $\varphi: M \rightarrow N$ between $n$ manifolds if $f_{1}$ and $f_{2}$ are $\varphi$-related then $A\left(f_{1}\right)$ and $A\left(f_{2}\right)$ are $T^{r *} \varphi$-related. The regularity means that $A$ transforms smoothly parametrized families of maps into smoothly parametrized families of affinors.

EXAMPLE 1 . For any $n$-manifold $M$ we have the identity affinor $\operatorname{Id}_{T T^{r *} M}$ : $T T^{r *} M \rightarrow T T^{r *} M$ over $T^{r *} M$. Hence we have the (constant) natural operator $\delta: T_{\mid \mathcal{M} f_{n}}^{(0,0)} \rightsquigarrow T^{(1,1)} T^{r *}$ such that $\delta(f)=\operatorname{Id}_{T T^{r *} M}$ for any map $f: M \rightarrow \mathbb{R}$ from an $n$-manifold $M$.

EXAMPLE 2. Let $p, q=0, \ldots, r$ be such that $1 \leq p+q \leq r$. Let $f: M \rightarrow \mathbb{R}$ be a map from an $n$-manifold $M$. We have an affinor

$$
A^{p, q}(f)=d f^{V} \otimes L^{p, q}(f)
$$

on $T^{r *} M$, where $f^{V}=f \circ \pi: T^{r *} M \rightarrow \mathbb{R}$ is the vertical lifting of $f$ to $T^{r *} M$ and $L^{p, q}$ is the vertical vector field on $T^{r *} M$ given by

$$
L^{p, q}(f)_{j_{x}^{r}(\gamma)}=\left(j_{x}^{r}(\gamma), j_{x}^{r}\left((f-f(x))^{p} \gamma^{q}\right)\right) \in\left\{j_{x}^{r}(\gamma)\right\} \times T_{x}^{r *} M \cong V_{j_{x}^{r}(\gamma)} T^{r *} M
$$

for $j_{x}^{r}(\gamma) \in T^{r *} M$. The correspondence $A^{p, q}: T_{\mid \mathcal{M} f_{n}}^{(0,0)} \rightsquigarrow T^{(1,1)} T^{r *}, f \mapsto$ $A^{p, q}(f)$, is a natural operator.

EXAMPle 3 . Let $p, q=0, \ldots, r$ be such that $1 \leq p+q \leq r$. Let $f: M \rightarrow \mathbb{R}$ be a map from an $n$-manifold $M$. We have an affinor $B^{p, q}(f)$ on $T^{r *} M$ such that

$$
B^{p, q}(f)_{j_{x}^{r}(\gamma)}=d_{j_{x}^{r}(\gamma)} \gamma^{V} \otimes L^{p, q}(f)_{j_{x}^{r}(\gamma)}
$$

for $j_{x}^{r}(\gamma) \in T^{r *} M$, where $\gamma^{V}=\gamma \circ \pi$ is the vertical lifting of $\gamma: M \rightarrow \mathbb{R}$ to $T^{r *} M$ and $L^{p, q}$ is the vertical vector field on $T^{r *} M$ as in Example 2. The correspondence $B^{p, q}: T_{\mid \mathcal{M} f_{n}}^{(0,0)} \rightsquigarrow T^{(1,1)} T^{r *}, f \mapsto B^{p, q}(f)$, is a natural operator. 
3. A classification of natural affinors on $T^{r *}$. Observe that $C^{q}:=$ $B^{0, q}(f)$ is a canonical affinor on $T^{r *} M$ (not depending on $f$ ). Similarly, $\delta$ is a canonical affinor on $T^{r *}$.

Let us quote a result of J. Kurek [5].

THEOREM 1. For natural numbers $n \geq 2$ and $r \geq 1$ the vector space of natural affinors on $T^{r *}$ is $(r+1)$-dimensional. More precisely, the natural affinors $\delta$ and $C^{q}$ for $q=1, \ldots, r$ form a basis over $\mathbb{R}$ in the vector space of all natural affinors on $T^{r *}$.

The following fact follows easily from Theorem 1 .

Corollary 1. Let $n \geq 2$ and $r \geq 1$ be natural numbers. Let $B$ be a natural affinor on $T^{r *}$ over $n$-manifolds. Suppose that $B\left(\partial_{1}^{C} \mid j_{0}^{r}\left(x^{1}\right)\right)=0$, where ( $)^{C}$ denotes the complete lifting (flow operator) of vector fields to $T^{r *}$. Then $B=0$.

4. The main result. The space of all natural operators $A: T_{\mathcal{M} f_{n}}^{(0,0)}$ $T^{(1,1)} T^{r *}$ is a module over $\mathcal{C}^{\infty}(\mathbb{R})$. Indeed, if $A: T_{\mathcal{M} f_{n}}^{(0,0)} \rightsquigarrow T^{(1,1)} T^{r *}$ is a natural operator and $g \in \mathcal{C}^{\infty}(\mathbb{R})$ then $g A: T_{\mid \mathcal{M} f_{n}}^{(0,0)} \rightsquigarrow T^{(1,1)} T^{r *}$ is given by $(g A)(f)=\left(g \circ f^{V}\right) A(f)$ for any $f: M \rightarrow \mathbb{R}$.

The main result of the present paper is the following classification theorem.

TheOREM 2. For natural numbers $n \geq 3$ and $r \geq 1$ the $\mathcal{C}^{\infty}(\mathbb{R})$-module of all natural operators $T_{\mid \mathcal{M} f_{n}}^{(0,0)} \rightsquigarrow T^{(1,1)} T^{r *}$ is free. More precisely, the $(r+1)(r+2)-1$ natural operators $\delta, A^{p, q}$ and $B^{p, q}$ for $p, q=0,1, \ldots, r$ with $1 \leq p+q \leq r$ form a basis in that $\mathcal{C}^{\infty}(\mathbb{R})$-module.

The proof of Theorem 2 will occupy Sections 5-9.

5. Beginning of proof of Theorem 2. From now on let $A: T_{\mid \mathcal{M} f_{n}}^{(0,0)} \rightsquigarrow$ $T^{(1,1)} T^{r *}$ be a natural operator, $n \geq 3$.

Let $\widetilde{A}: T_{\mid \mathcal{M} f_{n}}^{(0,0)} \rightsquigarrow T^{(1,1)} T^{r *}$ be the natural operator given by $\widetilde{A}(f)_{\mid T_{x}^{r *} M}$ $=A(f(x))_{\mid T_{x}^{r *} M}, x \in M$. If $k \in \mathbb{R}$ then $A(k)$ is a canonical affinor on $T^{r *}$. So, by Theorem 1, $\widetilde{A}$ is a linear combination of $\delta$ and $A^{0, q}$ with coefficients being maps $\mathbb{R} \rightarrow \mathbb{R}$. Hence on replacing $A$ by $A-\widetilde{A}$ we can assume that

$$
A(k)=0
$$

for any $k \in \mathbb{R}$.

We are going to show that $A$ is a linear combination of $A^{p, q}$ for $p=$ $1, \ldots, r$ and $q=0,1, \ldots$ with $p+q \leq r$ and $B^{p, q}$ for $p, q=0,1, \ldots$ with $1 \leq p+q \leq r$ with coefficient being maps $\mathbb{R} \rightarrow \mathbb{R}$. (We can easily see that 
$\delta, A^{p, q}$ and $B^{p, q}$ for $p, q=0,1, \ldots$ with $1 \leq p+q \leq r$ are $\mathcal{C}^{\infty}(\mathbb{R})$-linearly independent.)

\section{A reducibility lemma}

\section{Lemma 1. If}

$$
A\left(x^{1}+k\right)\left(\partial_{1}^{C} \mid j_{0}^{r}\left(x^{2}\right)\right)=A\left(x^{1}+k\right)\left(\partial_{2}^{C} \mid j_{0}^{r}\left(x^{2}\right)\right)=0
$$

and

$$
A\left(x^{1}+k\right)\left(\left.\frac{d}{d t}\right|_{t=0}\left(j_{0}^{r}\left(x^{2}\right)+t j_{0}^{r}\left(x^{3}\right)\right)\right)=0
$$

for any $k \in \mathbb{R}$, then $A=0$.

Proof. We have to show that $A(f)(w)=0$ for any $f: M \rightarrow \mathbb{R}$ and any $w \in T_{y} T^{r *} M$. By the naturality of $A$, the rank theorem and a density argument we can assume that $M=\mathbb{R}^{n}, f=x^{1}+k, y=j_{0}^{r}\left(x^{2}\right)$. Next, by the fiber linearity of $A(f)$ we can assume that $w=\partial_{\mid j_{0}^{r}\left(x^{2}\right)}^{C}$ or $w=$ $\left.\frac{d}{d t}\right|_{t=0}\left(j_{0}^{r}\left(x^{2}\right)+t j_{0}^{r} \gamma\right)$ for some constant vector field $\partial$ on $\mathbb{R}^{n}$ and some $\gamma$ : $\mathbb{R}^{n} \rightarrow \mathbb{R}$ with $\gamma(0)=0$. Then by the naturality of $A$, the fiber linearity of $A(f)$, the rank theorem and a density argument we can assume additionally that $\partial=\partial_{1}$ or $\partial=\partial_{2}$ and $\gamma=x^{3}$.

Lemma 1 shows that $A$ is determined by the values $A\left(x^{1}+k\right)\left(\partial_{1}^{C} \mid j_{0}^{r}\left(x^{2}\right)\right)$, $A\left(x^{1}+k\right)\left(\partial_{2}^{C} \mid j_{0}^{r}\left(x^{2}\right)\right)$ and $A\left(x^{1}+k\right)\left(\left.\frac{d}{d t}\right|_{t=0}\left(j_{0}^{r}\left(x^{2}\right)+t j_{0}^{r}\left(x^{3}\right)\right)\right)$ for any $k \in \mathbb{R}$.

\section{The verticality lemma}

Lemma 2. Under assumption (1), A is of vertical type, i.e. $\operatorname{im}(A(f)) \subset$ $V T^{r *} M$ for any $f: M \rightarrow \mathbb{R}$.

Proof. By Lemma 1 it is sufficient to show that the vectors

$$
\begin{gathered}
A\left(x^{1}+k\right)\left(\partial_{1 \mid j_{0}^{r}\left(x^{2}\right)}^{C}\right), \quad A\left(x^{1}+k\right)\left(\partial_{2 \mid j_{0}^{r}\left(x^{2}\right)}^{C}\right), \\
A\left(x^{1}+k\right)\left(\left.\frac{d}{d t}\right|_{t=0}\left(j_{0}^{r}\left(x^{2}\right)+t j_{0}^{r}\left(x^{3}\right)\right)\right)
\end{gathered}
$$

are vertical for any $k \in \mathbb{R}$.

We can write

$$
T \pi\left(A\left(x^{1}+k\right)\left(\partial_{1 \mid j_{0}^{r}\left(x^{2}\right)}^{C}\right)\right)=\sum_{i=1}^{n} a_{i}(k) \partial_{i \mid 0}
$$

for some $a_{i} \in \mathbb{R}$. Using the naturality of $B$ with respect to the homotheties $\tau \operatorname{id}_{\mathbb{R}^{n}}$ for $\tau \neq 0$ we obtain

$$
T \pi\left(A\left(\tau x^{1}+k\right)\left(\partial_{1 \mid j_{0}^{r}\left(\tau x^{2}\right)}\right)\right)=\sum_{i=1}^{n} a_{i}(k) \partial_{i \mid 0} .
$$


Letting $\tau \rightarrow 0$ and using (1) we see that $a_{1}(k)=\ldots=a_{n}(k)=0$. Then $A\left(x^{1}+k\right)\left(\partial_{1}^{C} \mid j_{0}^{r}\left(x^{2}\right)\right)$ is vertical.

Similarly we can proceed in the two remaining cases.

8. Some inessential assumptions. Let $i=1,2$. Because $A$ is of vertical type we can write

$$
A\left(x^{1}+k\right)\left(\partial_{i}^{C} \mid j_{0}^{r}\left(x^{2}\right)\right)=\left.\frac{d}{d t}\right|_{t=0}\left(j_{0}^{r}\left(x^{2}\right)+t j_{0}^{r}\left(\gamma_{i}(k, \cdot)\right)\right)
$$

for some $\gamma_{i}: \mathbb{R} \times \mathbb{R}^{n} \rightarrow \mathbb{R}$ with $\gamma_{i}(k, 0)=0$. By the naturality of $A$ with respect to $\left(x^{1}, x^{2}, \tau x^{3}, \ldots, \tau x^{n}\right)$ for $\tau \neq 0$ we have

$$
A\left(x^{1}+k\right)\left(\partial_{i}^{C} \mid j_{0}^{r}\left(x^{2}\right)\right)=\left.\frac{d}{d t}\right|_{t=0}\left(j_{0}^{r}\left(x^{2}\right)+t j_{0}^{r}\left(\gamma_{i}\left(k, x^{1}, x^{2}, \tau x^{3}, \ldots, \tau x^{n}\right)\right)\right) .
$$

Letting $\tau \rightarrow 0$ we have

$$
\begin{aligned}
& A\left(x^{1}+k\right)\left(\partial_{i \mid j_{0}^{r}\left(x^{2}\right)}^{C}\right) \\
& \quad=\left.\sum_{1 \leq p+q \leq r} a_{i, p, q}(k) \frac{d}{d t}\right|_{t=0}\left(j_{0}^{r}\left(x^{2}\right)+t j_{0}^{r}\left(\left(x^{1}\right)^{p}\left(x^{2}\right)^{q}\right)\right)
\end{aligned}
$$

for some smooth maps $a_{i, p, q}: \mathbb{R} \rightarrow \mathbb{R}$.

Next we use the invariance of $A$ with respect to $\left(\tau x^{1}, x^{2}, \ldots, x^{n}\right)$ for $\tau \neq 0$. From (4) we obtain

$$
\begin{aligned}
& A\left(\tau x^{1}+k\right)\left(\partial_{2}^{C} \mid j_{0}^{r}\left(x^{2}\right)\right) \\
& \quad=\left.\sum_{1 \leq p+q \leq r} \tau^{p} a_{2, p, q}(k) \frac{d}{d t}\right|_{t=0}\left(j_{0}^{r}\left(x^{2}\right)+t j_{0}^{r}\left(\left(x^{1}\right)^{p}\left(x^{2}\right)^{q}\right)\right) .
\end{aligned}
$$

Letting $\tau \rightarrow 0$ and using (1) we get

$$
a_{2,0, q}=0
$$

Then by replacing $A$ by

we can assume

$$
A-\sum_{1 \leq p+q \leq r} a_{1, p, q} B^{p, q}-\sum_{1 \leq p+q \leq r, p \neq 0} a_{2, p, q} A^{p, q}
$$

$$
A\left(x^{1}+k\right)\left(\partial_{1}^{C} \mid j_{0}^{r}\left(x^{2}\right)\right)=A\left(x^{1}+k\right)\left(\partial_{2}^{C} \mid j_{0}^{r}\left(x^{2}\right)\right)=0
$$

for any $k \in \mathbb{R}$.

9. End of proof of Theorem 2. Given an $(n-1)$-manifold $N$ we have the obvious inclusion $T^{r *} N \subset T^{r *}(\mathbb{R} \times N)$ given by $j_{z}^{r} \gamma \mapsto j_{(0, z)}^{r} \gamma$, $\gamma: N \rightarrow \mathbb{R}, z \in N, \gamma(z)=0$, where we identify $\gamma$ with $\gamma \circ \operatorname{pr}_{N}, \operatorname{pr}_{N}$ : $\mathbb{R} \times N \rightarrow N$ being the projection. Then for any $j_{z}^{r} \gamma \in T^{r *} N$ we have the induced inclusion $T_{j_{z}^{r} \gamma} T^{r *} N \subset T_{j_{(0, z)}^{r} \gamma} T^{r *}(\mathbb{R} \times N)$. 
For $q=0, \ldots, r$ we define an $\mathcal{M} f_{n-1}$-natural affinor $B_{q}: T T^{r *} N \rightarrow$ $T T^{r *} N$ on $T^{r *} N$ as follows. Let $w \in T_{j_{z}^{r} \gamma} T^{r *} N, \gamma: N \rightarrow \mathbb{R}, z \in N$, $\gamma(z)=0$. Then $w \in T_{j_{(0, z)}^{r} \gamma} T^{r *}(\mathbb{R} \times N)$ and we can apply $A\left(x^{1}+k\right)$ to $w$, where $x^{1}: \mathbb{R} \times N \rightarrow \mathbb{R}$ is the projection. We have the elements $j_{z}^{r-q}\left(\eta_{q}^{w}\right) \in$ $J_{z}^{(r-q) *}(N, \mathbb{R})\left(\eta_{q}^{w}: N \rightarrow \mathbb{R}, \eta_{0}^{w}(z)=0\right)$ linearly depending on $w$ by

$$
A\left(x^{1}+k\right)(w)=\left.\frac{d}{d t}\right|_{t=0}\left(j_{(0, z)}^{r} \gamma+t j_{(0, z)}^{r}\left(\sum_{q=0}^{r}\left(x^{1}\right)^{q} \eta_{q}^{w}\right)\right) .
$$

We put

$$
B_{q}(w)=\left.\frac{d}{d t}\right|_{t=0}\left(j_{z}^{r} \gamma+t j_{z}^{r}\left(\gamma^{q} \eta_{q}^{w}\right)\right) .
$$

From (7) we deduce that $B_{q}\left(\partial_{2}^{C} \mid j_{0}^{r}\left(x^{2}\right)\right)=0$, where we identify $x^{2}, \ldots, x^{n}$ with the usual coordinates on $\mathbb{R}^{n-1}=\{0\} \times \mathbb{R}^{n-1}$. Then by Corollary 1 we have $B_{q}=0$ for $q=0, \ldots, r$. Hence (in particular)

$$
A\left(x^{1}+k\right)\left(\left.\frac{d}{d t}\right|_{t=0}\left(j_{0}^{r}\left(x^{2}\right)+t j_{0}^{r}\left(x^{3}\right)\right)\right)=0,
$$

and thus $A=0$ because of Lemma 1 .

10. Corollaries. Let $L(f)$ be a vector field on $T^{r *} M$ canonically depending on a function $f: M \rightarrow \mathbb{R}$. Then $d f^{V} \otimes L(f)$ is an affinor on $T^{r *} M$ canonically dependent on $f$. Using Theorem 2 we can write $d f^{V} \otimes L(f)$ as a linear combination of $A^{p, q}(f)$ and $B^{p, q}(f)$ with coefficients from $\mathcal{C}^{\infty}(\mathbb{R})$. Then it is easy to verify that $L(f)$ is a linear combination of $L^{p, q}(f)$ with coefficients from $\mathbb{R}$. Thus we get the following fact.

COROLlaRY 2. For natural numbers $n \geq 3$ and $r \geq 1$ the natural operators $L^{p, q}$ for $p, q=0,1, \ldots, r$ with $1 \leq p+q \leq r$ form a basis over $\mathbb{R}$ in the vector space of all natural operators $T_{\mid \mathcal{M} f_{n}}^{(0,0)} \rightsquigarrow T T^{r *}$.

Let $\Omega(f)$ be a vector 1-form on $T^{r *} M$ canonically depending on a function $f: M \rightarrow \mathbb{R}$. Then $\Omega(f) \otimes L^{0,1}$ is an affinor on $T^{r *} M$ canonically dependent on $f$. Using Theorem 2 we can write $\Omega(f) \otimes L^{0,1}$ as a linear combination of $A^{p, q}(f)$ and $B^{p, q}(f)$ with coefficients from $\mathcal{C}^{\infty}(\mathbb{R})$. Then it is easy to verify that $\Omega(f)$ is a linear combination of $d f^{V}$ and $\lambda^{r}$ with coefficients from $\mathcal{C}^{\infty}(\mathbb{R})$, where $\lambda^{r}$ is the usual canonical 1-form on $T^{r *} M$. Thus we get the following fact.

COROLlary 3. For natural numbers $n \geq 3$ and $r \geq 1$ the natural operators $d f^{V}$ and $\lambda^{r}$ form a basis over $\mathcal{C}^{\infty}(\mathbb{R})$ in the $\mathcal{C}^{\infty}(\mathbb{R})$-module of all natural operators $T_{\mid \mathcal{M} f_{n}}^{(0,0)} \rightsquigarrow T^{*} T^{r *}$. 


\section{References}

[1] M. Doupovec and I. Kolář, Natural affinors on time-dependent Weil bundles, Arch. Math. (Brno) 27 (1991), 205-209.

[2] J. Gancarzewicz and I. Kolár, Natural affinors on the extended rth order tangent bundles, Rend. Circ. Mat. Palermo 30 (1993), 95-100.

[3] I. Kolár̆, P. W. Michor and J. Slovák, Natural Operations in Differential Geometry, Springer, Berlin, 1993.

[4] I. Kolář and M. Modugno, Torsions of connections on some natural bundles, Differential Geom. Appl. 2 (1992), 1-16.

[5] J. Kurek, Natural affinors on higher order cotangent bundle, Arch. Math. (Brno) 28 (1992), 175-180.

[6] W. M. Mikulski, Natural affinors on r-jet prolongation of the tangent bundle, ibid. 34 (1998), 321-328.

[7] - Natural affinors on $\left(J^{r, s, q}\left(\cdot, \mathbb{R}^{1,1}\right)_{0}\right)^{*}$, Comment. Math. Univ. Carolin. 42 (2001), 655-663.

Institute of Mathematics

Jagiellonian University

Reymonta 4

30-059 Kraków, Poland

E-mail: mikulski@im.uj.edu.pl 